Published in final edited form as:

Eur J Haematol. 2020 January ; 104(1): 72-73. doi:10.1111/ejh.13335.

\title{
Outcomes of Splanchnic Vein Thrombosis in Patients with Myeloproliferative Neoplasms in a Single Center Experience
}

\author{
Douglas Tremblay ${ }^{1}$, Alexander S. Vogel ${ }^{2}$, Erin Moshier ${ }^{3}$, Ronald Hoffman ${ }^{1}$, Marina \\ Kremyanskaya $^{1}$, Selena Zhou ${ }^{1}$, Thomas Schiano ${ }^{2}$, John Mascarenhas ${ }^{1}$ \\ ${ }^{1}$ Tisch Cancer Institute, Icahn School of Medicine at Mount Sinai, New York, NY \\ 2Division of Liver Diseases, Recanati/Miller Transplantation Institute, Icahn School of Medicine at \\ Mount Sinai, New York, NY; \\ ${ }^{3}$ Department of Population Health Science and Policy/Tisch Cancer Institute, Icahn School of \\ Medicine at Mount Sinai, New York
}

\section{Keywords}

Splanchnic vein; thrombosis; myeloproliferative; direct oral anticoagulant

To the Editor:

Myeloproliferative neoplasms (MPNs) are a group of hematologic malignancies characterized by overlapping clinical features including an increased thrombotic potential. ${ }^{1}$ Splanchnic vein thrombosis (SVT) has a known association with MPNs, in which $8.2 \%$ of SVTs occur in the setting of an underlying MPN. ${ }^{2}$ A main consequence of SVT is portal hypertension, which can lead to complications including esophageal variceal bleeding (EVB) and ascites. ${ }^{3}$ The impact of treating the underlying MPN at time of SVT on these hepatic outcomes is unknown.

We conducted a retrospective cohort study of 64 patients with MPNs who experienced an SVT between January 1995 and April 2018. Timing of SVT occurrence relative to MPN diagnosis was determined to be concurrent if SVT was diagnosed within 30 days of MPN. EVB and complications of anticoagulation were defined as International Society on Thrombosis and Haemostasis major bleeding. ${ }^{4}$ We used a 6-month landmark survival analysis for outcomes in order to account for immortal time bias arising from varying times to MPN diagnosis following SVT.

Table 1 details the characteristics of the cohort. SVT was diagnosed a median of 4 [1-90] months prior to diagnosis of MPN in the SVT antecedent to MPN diagnosis group while SVT was diagnosed a median of 46 [1-288] months after diagnosis of MPN in the SVT after MPN diagnosis group.

Correspondence: John Mascarenhas, MD, Associate Professor of Medicine, Myeloproliferative Disorders Program, Tisch Cancer Institute, Division of Hematology/Oncology, Icahn School of Medicine at Mount Sinai, One Gustave L Levy Place, Box 1079, New York, New York 10029, office: 212241 3417, fax: 2128765276. 
During a median follow-up of 61 months from time of SVT (1 to 279 months), EVB occurred in 13 (20\%) patients, ascites in 30 patients (47\%), and hepatic encephalopathy in 6 patients (9\%). There was no difference between patients who were receiving MPN treatment at time of SVT diagnosis and those who did not for time to EVB $(\mathrm{p}=0.3519)$, additional SVT $(\mathrm{p}=0.6995)$, ascites $(\mathrm{p}=0.8016)$, or hepatic encephalopathy $(\mathrm{p}=0.2642)$. There was no difference in time to composite of these outcomes between those receiving MPN-direct therapy at time of SVT diagnosis versus patients who were not $(\mathrm{p}=0.8983)$ or in patients who were receiving anticoagulation and those who were not $(\mathrm{p}=0.9222)$. The median OS of the cohort was not reached. The five-year OS probability was $98 \%$ and there was no difference among patients diagnosed antecedent, concurrent, or after MPN diagnosis. Twelve patients (25\% of those anticoagulated) were treated with a direct oral anticoagulant (DOAC) for median duration of 23.9 [1.7-77.6] months. Only one patient experienced a bleeding event and one patient developed recurrent thrombosis while receiving a DOAC.

Our study has a number of limitations that are important to recognize. Most importantly, our small sample size limited our ability to compare subgroups. The study period encompasses evolving diagnostic and treatment paradigms for both MPNs and SVTs. Finally, laboratory values prior to thrombosis, including gamma-glutamyl transferase, were inconsistently available, limiting our ability to confirm prior studies linking lab values to development of SVT. $^{5}$

In conclusion, we provide new information regarding the sequencing of SVT as it relates to MPN diagnosis. In a small subgroup, DOACs appear to be safe in the treatment of MPNassociated SVT. We were not able to show dependence between treatment of the underlying MPN at time of SVT and rates of EVB, additional SVT, development of ascites, and hepatic encephalopathy. Future studies are required to further clarify the optimal therapeutic strategies for patients with an MPN-associated SVT.

\section{References}

1. Falanga A, Marchetti M. Thrombotic disease in the myeloproliferative neoplasms. Hematology Am Soc Hematol Educ Program. 2012;2012:571-581. [PubMed: 23233637]

2. Ageno W, Riva N, Schulman S, et al. Long-term Clinical Outcomes of Splanchnic Vein Thrombosis: Results of an International Registry. JAMA Intern Med. 2015;175(9):1474-1480. [PubMed: 26168152]

3. Lavu S, Szuber N, Mudireddy M, et al. Splanchnic vein thrombosis in patients with myeloproliferative neoplasms: The Mayo clinic experience with 84 consecutive cases. Am J Hematol. 2018;93(3):E61-E64. [PubMed: 29194707]

4. Schulman S, Kearon C, Subcommittee on Control of Anticoagulation of the S, Standardization Committee of the International Society on T, Haemostasis. Definition of major bleeding in clinical investigations of antihemostatic medicinal products in non-surgical patients. J Thromb Haemost. 2005;3(4):692-694. [PubMed: 15842354]

5. Gortzen J, Hunka LM, Vonnahme M, et al. gamma-Glutamyl Transferase Is an Independent Biomarker of Splanchnic Thrombosis in Patients With Myeloproliferative Neoplasm. Medicine (Baltimore). 2016;95(20):e3355. [PubMed: 27196445] 
Table 1.

\section{Patient Characteristics}

\begin{tabular}{|c|c|}
\hline Subjects, N & 64 \\
\hline Age at MPN diagnosis, years median (range) & 43 [16-89] \\
\hline Age at Thrombosis, years median (range) & 45 [18-89] \\
\hline Year of MPN diagnosis, median (range) & 2010 [1981-2018] \\
\hline Female, N (\%) & $46(72 \%)$ \\
\hline \multicolumn{2}{|l|}{ MPN Diagnosis, N (\%) } \\
\hline PMF & $8(13 \%)$ \\
\hline PV & $29(45 \%)$ \\
\hline ET & $14(22 \%)$ \\
\hline Post ET/PV MF & $5(8 \%)$ \\
\hline Pre-PMF & $2(3 \%)$ \\
\hline MDS/MPN, unclassifiable & $1(2 \%)$ \\
\hline MPN unclassifiable & $5(8 \%)$ \\
\hline \multicolumn{2}{|l|}{ Mutational status, $\mathrm{N}(\%)^{*}$} \\
\hline$J A K 2$ & $60(95 \%)$ \\
\hline$C A L R$ & $1(1.6 \%)$ \\
\hline DMNT3A & $1(1.6 \%)$ \\
\hline$E Z H 2$ & $1(1.6 \%)$ \\
\hline \multicolumn{2}{|l|}{ Timing of thrombosis, $\mathrm{N}(\%)$} \\
\hline After MPN & $28(44 \%)$ \\
\hline Antecedent to MPN & $19(30 \%)$ \\
\hline Concurrent & $17(26 \%)$ \\
\hline Multiple thromboses, N (\%) & $23(36 \%)$ \\
\hline \multicolumn{2}{|l|}{ Thrombosis type, $\mathrm{N}(\%)$} \\
\hline BCS & $23(36 \%)$ \\
\hline PVT & $44(69 \%)$ \\
\hline Other & $23(36 \%)$ \\
\hline Splenic Vein Thrombosis & $13(20 \%)$ \\
\hline Superior Mesenteric Vein Thrombosis & $9(14 \%)$ \\
\hline IVC & $1(2 \%)$ \\
\hline Portal vein cavernous transformation, $\mathrm{N}(\%)$ & $11(33 \%)$ \\
\hline \multicolumn{2}{|l|}{ MPN treatment at thrombosis, $\mathrm{N}(\%)$} \\
\hline Hydroxyurea & $3(5 \%)$ \\
\hline Interferon-alpha & $3(5 \%)$ \\
\hline Ruxolitinib & $1(2 \%)$ \\
\hline Anagrelide & $1(2 \%)$ \\
\hline Therapeutic Phlebotomy Only & $3(5 \%)$ \\
\hline
\end{tabular}

Eur J Haematol. Author manuscript; available in PMC 2021 January 04. 


\begin{tabular}{ll} 
Lestaurtinib & $1(2 \%)$ \\
\hline None & $44(69 \%)$ \\
\hline Unknown & $8(13 \%)$ \\
\hline Treatment of SVT, N $(\%)$ & $12(19 \%)$ \\
\hline Enoxaparin & $25(39 \%)$ \\
\hline Warfarin & $8(13 \%)$ \\
\hline Aspirin & $12(19 \%)$ \\
\hline DOAC & $8(13 \%)$ \\
\hline Rivaroxaban & $3(5 \%)$ \\
\hline Dabigatran & $1(2 \%)$ \\
\hline Apixaban & $13(20 \%)$ \\
\hline Transjugular intrahepatic portosystemic shunt & $7(11 \%)$ \\
\hline None & $2(3 \%)$ \\
\hline Liver Transplantation & $1(2 \%)$ \\
\hline Other Surgical Procedure & $2(3 \%)$ \\
\hline Unknown & $1(2 \%)$ \\
\hline Fondaparinux & \\
\hline
\end{tabular}

BCS - Budd Chiari syndrome; DOAC = direct oral anti-coagulant;

ET - essential thrombocythemia; IVC = inferior vena cava;

MDS - myelodysplastic syndrome; MPN - myeloproliferative neoplasms;

PMF - primary myelofibrosis; PV - polycythemia vera;

PVT - portal vein thrombosis; SVT - splanchnic vein thrombosis

Non-driver mutational analysis was only performed on 9 patients (14\%) at diagnosis 